\title{
NOTARIATO MILANESE E NOTARIATO ARETINO MEDIEVALI A CONFRONTO (SECOLI XIII-XIV)
}

\author{
MEDIEVAL NOTARIES IN MILAN AND AREZZO: A COMPARISON \\ (13TH-14TH CENTURIES)
}

\author{
Gian Paolo Giuseppe Scharf
}

Dottore di ricerca Università di Perugia

\begin{abstract}
English: Approaching notarial production and uses in two different Italian communal cities, during the last medieval centuries, is a major way of highlighting specific declinations of a unique notarial civilization, that of Italian communal city-states. Considering the cities and their territories ("contadi") as a whole, the essay first of all stresses the contadi sizes, their populations, the presence of towns and small villages, seen as the basical action field for the respective notaries. The second step consists in evaluating how much of the impressive notarial production for the two studied centuries is actually stored in the respective archives. Relatively known is the medieval documentation abundant loss trough the centuries, but the essay tries to estimate the remaining actual percentage, stressing the attention over the diplomatic sources weight, i.e. the parchments, wich form a particular type of notarial production, but nevertheless noteworthy.
\end{abstract}

The third point of the article push forward an analysis of notarial careers, noting how much urban-centered they were in the two studied cities, more in Arezzo than in Milan. Is useful observing that the "contado" of the second was really full of notarial dinasties, scattered through the territory, not only in the Milanese little towns ("borghi"), but also in modest villages. The most important information concerns the different degrees of notarial career: in Arezzo only a step was considered enough, while in Milan normally notaries went trough a three step path: beginning with pronotarius, the professionist became notarius (laudatus ad omnia) and then iudex ordinarius. The complete unfolding of this path, nevertheless, is given only since the XIV century second half, when is possible to see it more frequently.

The essay last part focuses about the notarial formulary, which reveals less differences than expected between the two cities. Among a common set of uses and practices two minor discrepancies catch the attention: Milanese notaries indicated very often a clause about the use prohibition of communal "carte debiti", a sort of civic money substitute. That clause is normally absent in documents drawn up in Arezzo, revealing a different urban financing system. On the other hand the latter's documents contain nearly always a different clause about the "preceptum guarentigie", a communal law which permits immediate use of notarial debt documents, without court intervention. That clause also reveals a different debt legislation between the two cities.

Nevertheless this two examples confirm the existence of a unique notarial civilization in Arezzo and Milan, declined somehow differently.

* Italian Review of Legal History, 7 (2021), n. 20, pagg. 677-689

* https://riviste.unimi.it/index.php/irlh/index

* ISSN 2464-8914 - DOI 10.54103/2464-8914/16905. Articolo pubblicato sotto Licenza CC-BY. 
Keywords: Notarial Civilization; Communal Period; Arezzo; Milan; Notarial Careers

Abstract Italiano: Milano e Arezzo, due città apparentemente molto distanti, ma in realtà piuttosto simili in fatto di civiltà notarile, vengono qui confrontate da questo punto di vista per evidenziare similitudini e particolarità della produzione notarile, della sua conservazione, della carriera e del ruolo di tali protagonisti, del formulario usato da essi. L'analisi viene estesa anche al contado, considerato parte inscindibile di un unico spazio comunale che nella città aveva il suo centro.

Parole chiave: Civiltà notarile; epoca comunale; Arezzo; Milano; carriere notarili

Sommario: 1. Milano e Arezzo, due città comunali - 2. Il territorio milanese e quello aretino, come campo d'azione del notariato - 3. La documentazione notarile milanese e aretina - 4. II notariato nelle due città: ambiti di attività e carriere - 5. II formulario notarile nelle due città.

\section{Milano e Arezzo, due città comunali}

Nel panorama delle città medievali e dei loro contadi Milano e Arezzo sono indubbiamente molto diverse. E tuttavia le due città hanno anche in comune non poche cose, la principale delle quali è bene ricordare e non dare per scontata. Entrambe, infatti, appartengono teoricamente al Regnum Italie, cosa che significa partecipare di quello stesso milieu culturale comunale che favorisce sviluppi simili anche in regioni differenti. Tale milieu culturale è poi il miglior brodo di coltura della civiltà notarile, non dimentichiamolo, poiché le caratteristiche del notariato italiano sono intrinsecamente legate alla civiltà comunale ${ }^{1}$.

In effetti, dato che qui si tenterà di effettuare un confronto - speriamo proficuo - fra le due differenti declinazioni del notariato medievale italiano, confronto basato essenzialmente sulla nostra esperienza empirica frutto di anni spesi negli archivi, non sarà fuori luogo definire prima l'oggetto di codesto confronto. Non ci soffermeremo però su una liminare definizione di notariato medievale italiano, sia perché argomento abbastanza noto nelle sue generalità (a dispetto delle molteplici sfumature assunte), sia perché durante il corso on line dal quale prende le mosse questo contributo innumeri interventi hanno sviscerato e acclarato tale definizione. Occorrerà invece spendere qualche parola sugli altri parametri di questa operazione, quelli cioè spaziali e temporali, visto che, come vedremo, hanno importanza non secondaria nell'orientare l'indagine 2 .

L'ovvia premessa allo studio è che il nostro orizzonte è urbano e rurale insieme,

1 La bibliografia sullo spazio comunale italiano, in quanto civiltà notarile, è sterminata e non si può qui dettagliatamente indicare. Si inizi comunque col celebre Pini, 1987; si vedano poi Ginatempo, 1997, Jones, 1997.

2 Inutile dire che anche sul notariato italiano si rischia di perdersi nella pletora di studi dedicati all'argomento. Per limitarci a cose recenti possiamo citare Menant, Redon (eds.), 
vale a dire che prende in considerazione l'unità - anche ideologica - di città e contado, così come erano definiti nell'epoca che ci interessa. Le affinità iniziano a manifestarsi al confronto di due contadi molto vasti, pedologicamente molto vari e non totalmente coincidenti con le rispettive diocesi, alle quali pure facevano riferimento. In effetti si trattava di due diocesi molto estese, fra le più vaste d'Italia, e tuttavia integrate anche da altri territori ${ }^{3}$.

\section{II territorio milanese e quello aretino, come campo d'azione del nota- riato}

La diocesi medievale di Milano copre quattro attuali provincie (Milano, Varese, Lecco e Monza), qualche centro della provincia comasca e una parte del Canton Ticino, senza contare la sponda piemontese del Lago Maggiore. Ma a questo spazio già enorme si aggiungeva l'incorporazione al contado milanese (dal punto di vista amministrativo) di territori facenti parte di altre diocesi: è il caso di non poche enclaves pavesi, situate soprattutto nell'attuale provincia varesina, come pure di un intero territorio pievano (e parte di un altro) della diocesi comasca, che si protendevano nel mezzo della diocesi milanese (sempre a nord di Varese) ed erano perciò considerati contado di Milano. Solo poche terre poste sul caldo confine pavese in certi periodi non fecero parte del contado milanese, pur essendo nella diocesi ambrosiana. Proprio per questo motivo il vastissimo territorio fu precocemente suddiviso amministrativamente in quattro "sottocontadi" (i più famosi sono il Seprio e la Martesana), ma ciò non ebbe alcuna rilevanza per usi e tradizioni notarili, che appaiono unitari per tutto il Medioevo ${ }^{4}$.

Anche Arezzo aveva, come abbiamo detto, una vasta diocesi, ma il contado non vi corrispondeva: il fenomeno singolare che si osserva agevolmente è lo spostamento verso est del contado aretino rispetto alla diocesi, fenomeno del resto che si riscontra anche oggi nella delimitazione della provincia. In pratica le fertili zone occidentali, fra Chianti e Valdichiana, gravitavano su Siena, per via dell'aggressiva espansione territoriale della città della Balzana, ma per converso nel territorio aretino rientrava quasi tutta la Valtiberina superiore facente parte della diocesi tifernate (e alcuni centri limitrofi di altre diocesi). Inoltre, l'intero Casentino era considerato territorio aretino, pur essendo parzialmente in diocesi di Fiesole. L'unica sfumatura della valle corrispondeva alla zona controllata dai Guidi, che

2004; Pinto, Tanzini, Tognetti (eds.), 2018. Sempre validi, comunque, gli studi di Fissore: Fissore, 1978; Fissore, 1989.

3 II particolare rapporto fra città comunale e contado è naturalmente al centro di molti studi sui comuni italiani. Oltre agli studi citati supra, nota 1 , vedi anche Chiappa Mauri (ed.), 2004; Mucciarelli, Piccinni, Pinto (eds.), 2009; Città e campagna nei secoli altomedievali, 2009; Chittolini, Willoweit (eds.), 1994.

4 Sul contado milanese vedi Chiappa Mauri, 1993, Gamberini, 2004, e sulla distrettuazione Riboldi, 1904, Castelnuovo, 2007; Scharf, 2015. 
dell'aretino faceva parte solo nominalmente. Anche in questo caso dobbiamo notare che l'unitarietà della civiltà notarile aretina corrispondeva al suo contado, non già alla sua diocesi $i^{5}$.

I due contadi così delineati erano diversamente popolati: il milanese letteralmente pullulava di borghi, diventati poi città (e alcuni attualmente provincia) ma in ogni caso di notevole spessore: è il caso perlomeno di Monza, Lecco, Treviglio, Varese, Gallarate, Busto Arsizio, centri con una tale vitalità da far pensare a "quasi città", come ha felicemente argomentato Chittolini. Non mancavano però numerosi centri minori, diffusi anche nelle più inospitali aree montane. L'aretino invece presentava una notevole diversificazione fra le quattro conche vallive che ne costituivano il territorio: la Valtiberina a est presentava soprattutto grossi centri, anche se non mancavano villaggi più minuti, soprattutto nella zona mineraria dei Monti Rognosi; la Valdichiana, a sud, essendo soggetta a frequenti impaludamenti, era praticamente priva di centri piccoli e il popolamento si addensava in alcuni grossi centri situati sulle sommità collinari (in un caso, Cortona, diventato poi città); la piana di Arezzo e il primo tratto del Valdarno, a ovest, essendo fertili e traversati da una viabilità esuberante, erano disseminati di piccoli centri, ma ne mancavano di più grandi; il Casentino, infine, a nord, presentava un fitto reticolo di piccoli villaggi e centri di più grosse dimensioni. Tutto ciò aveva delle evidenti ricadute per quanto riguarda la presenza e l'attività dei notai, anche se la corrispondenza non è così automatica come si potrebbe pensare ${ }^{6}$.

\section{La documentazione notarile milanese e aretina}

Le coordinate cronologiche dello studio rivelano la vera differenza fra le due situazioni quanto a consistenza della documentazione notarile.

A Milano il notarile, conservato in Archivio di Stato, comincia col 1347 (anche se esiste un registro precedente, conservato in un altro fondo) e comunque fino alla fine del secolo è discontinuo e poco consistente, per via delle immense perdite subite: è stato calcolato, in studi dedicati al notariato rurale, che quanto rimasto raggiunga a fatica l' $1 \%$ della produzione complessiva. II notarile aretino è invece conservato in buona parte presso l'Archivio di Stato di Firenze, ma sono rimasti in città proprio i pezzi più risalenti. Esiste dunque un intero registro e alcuni frammenti di documentazione duecentesca, mentre dal 1306 i registri presenti nell'archivio fiorentino si infittiscono, arrivando a diverse decine. Se vogliamo limitarci al Trecento, per poter effettuare una comparazione, possiamo avanzare una stima personale, che valuta al $10 \%$ della produzione quanto conservato. Le

\footnotetext{
5 L'ampia monografia del Delumeau dedicata ad Arezzo ne descrive accuratamente il contado, ma per un periodo precedente: Delumeau, 1996. Per il Duecento vedi Scharf, 2013. Qualche spunto anche in Dini, 1984; Dini, 1995; Canaccini, 2002.

${ }^{6}$ Sul popolamento in area milanese vedi ancora Chiappa Mauri, 1993, e Scharf, 2018a. Per l'area aretina vedi Scharf, 2013, e Barlucchi, 2013.
} 
due cifre, per quanto da prendere con molta cautela, danno già un senso generale alle nostre osservazioni ${ }^{7}$.

È opportuno aggiungere che la mancanza di un fondo notarile specifico, non si traduce automaticamente nell'assenza di documentazione di produzione notarile, soprattutto per il Duecento: possiamo trascurare i fondi pubblici, ugualmente andati perduti in massima parte nelle due città, ma concentrandoci sui fondi diplomatici conservati, la stima delle pergamene private disponibili è imponente. Per Arezzo limitandoci al Duecento, secolo di produzione più abbondante rispetto al seguente, si ha a che fare con forse qualcosa di più di cinquemila pergamene, mentre per Milano la stima della consistenza complessiva consiste in qualche centinaio di meno. Un'altra differenza di rilievo è data dal fatto che ad Arezzo i principali fondi pergamenacei appartengono a enti urbani (con la vistosa eccezione di Camaldoli), che però illuminano anche il contado, grazie ai loro vastissimi possessi. A Milano, invece, numerosi sono i fondi di enti non urbani, se non schiettamente rurali perlomeno situati nei borghi di cui abbiamo detto. Spiccano i fondi di due pievi del contado, Varese e Vimercate, che non hanno sostanzialmente paragoni con enti simili non solo nell'aretino, ma neanche nel milanese. Però appunto una era situata in un borgo, poi divenuto città (Varese) ${ }^{8}$.

\section{II notariato nelle due città: ambiti di attività e carriere}

Le caratteristiche del notariato, così come appare dalla documentazione rimasta, mostrano che la mobilità assoluta di tale ceto di professionisti era piuttosto scarsa, anche se discendeva da due ragioni differenti: a Milano perché i notai si trovavano un po' dovunque, mentre ad Arezzo perché la centralizzazione urbana dell'attività notarile era fortissima e i clienti si recavano in città anche da luoghi Iontani. È probabile che in entrambi i casi fra il notariato urbano non si dessero vere e proprie specializzazioni, almeno prima del Quattrocento, dato che i notai

\footnotetext{
7 Riguardo al notarile milanese la bibliografia non è poca: si inizi da Cereghini, 1992; Liva, 1979. I molti lavori della Mangini si sono concentrati soprattutto sul primo periodo (e sul primo pezzo conservato): vedi Mangini, 2011a; Mangini, 2011b; Mangini (ed.), 2011c; Mangini, 2012. Un censimento dei notai del contado in Lunari, Sala, Scharf (eds.), 2009. Per il solo territorio lecchese Guzzi, Mainoni, Zelioli Pini (eds.), 2012. Sul notarile aretino invece Franceschi, 2009; Scharf, 2018b.

8 Anche in questo caso la bibliografia potrebbe spaventare per la sua abbondanza: per Arezzo vedi Pasqui, 1916; Schiaparelli, Baldasseroni, Lasinio (eds.), 1909, 1914, 1922; Delumeau, 1996; Scharf, 2013. Per Milano le edizioni non si contano: a parte Varese, per la quale si vedano Merati (ed.), 2005, Merati (ed.), 2006, Merati (ed.), 2009, Perelli Cippo (ed.), 1976, Zagni (ed.), 1992, Zagni (ed.), 1999, Zagni (ed.), 2005; e Vimercate, per la quale si veda Fois (ed.), 2008, Fois (ed.), 2010, Fois (ed.), 2012, indispensabile il rimando a Baroni (ed.), 1976, Baroni (ed.), 1992, Baroni (ed.), 1997-1998, Baroni, Perelli Cippo (eds.), 1982-1988. Per la produzione diplomatica di un territorio, che può fungere da esempio, vedi Lucioni, Scharf (eds.), 2021.
} 
cittadini si occupavano di clienti un po' di tutti generi e di tutte le provenienze; questa osservazione può pure essere estesa al contado, a patto di limitarla alle tipologie negoziali, dato che nei piccoli centri i notai avevano clientela eminentemente locale, come è intuitivo. Ciò non vuol dire, come è ugualmente intuitivo, che ciascun professionista non avesse una clientela affezionata, che si rivolgeva prevalentemente a lui, solamente che non è dato riscontrare specializzazioni paragonabili a quelle del Quattrocento, quando a Milano sono attestati notai fissi per la camera dei Mercanti, o per qualche arte maggiore, circostanza che si presenta in misura minore ad Arezzo. A Milano, del resto, è presente un fondo (Rogiti Camerali) che raccoglie la documentazione di notai legati all'amministrazione signorile, ma almeno per il Trecento la loro attività non è esclusiva per essa9.

Il problema che si presenta a questo punto è assai importante e sarebbe opportuno approfondirlo: si tratta dei notai organici alle istituzioni, dato che il rapporto con strutture pubbliche ormai sviluppate e bisognose dell'apporto di numerosi professionisti può illuminare più di un aspetto del notariato. Tuttavia, anche in questo caso un legame ufficiale o perlomeno stabile non si dà prima del Quattrocento, pur essendo ancora da fare un'indagine a tutto campo nell'ambito delle istituzioni civili. L'altra istituzione di vertice dell'epoca, quella episcopale, sulla quale ricerche sono state compiute, non presenta una realtà molto diversa: certamente ci furono notai che rogarono prevalentemente per i vescovi o i capitoli delle due città, ma non è dato osservare vere e proprie specializzazioni nel periodo in esame. Un esempio e contrario proverà l'assunto: nella seconda metà del XIII secolo ad Arezzo fu molto attivo un notaio che rogò tanto per il vescovo, quanto per il capitolo (nella cui documentazione sono conservati i suoi registri), ma non tralasciò nel frattempo la clientela privata, al punto che quando finalmente venne promosso canonico egli stesso smise di rogare ${ }^{10}$.

L'origine dei notai del contado è certamente un punto di divaricazione fra le due realtà. Come abbiamo detto ad Arezzo i notai attivi nel contado sono molto pochi e appaiono figure isolate. Fanno eccezione i notai attivi per i conti Guidi, che ebbero anche clientela privata, pur essendo in genere assorbiti dal servizio comitale. Ma il territorio guidingo faceva parte del contado aretino solo formalmente, godendo di una sostanziale autonomia. Perciò si può dire che il resto dell'aretino mostrava un'omogenea facies di atonia notarile rurale. Non così nel territorio ambrosiano, dove si danno vere e proprie dinastie, attive per decenni, e ciò non solo nei borghi più grossi, ma anche nei paesi più piccoli: un esempio è dato dalla famiglia Scappi (o Scappa) di Dumenza, piccolo centro sulle montagne

\footnotetext{
9 Sui notai milanesi e le loro procedure vedi Baroni, 1978; per il periodo successivo Zagni, 1982; Bedina, 1992; per i notai attivi per la Camera dei Mercanti Del Bo, 2010. Sui notai che ad Arezzo lavorarono per i mercanti vedi Cherubini, 2001; Pinto, 1999.

10 Sui notai operanti per i vescovi in generale si veda Chittolini G., 1994. A Milano è stato fatto un censimento per l'età viscontea: Belloni, Lunari (eds.), 2004. Niente di simile per Arezzo, ma qualcosa si può desumere da Pieri, 2002, e Scharf, 2013.
} 
sopra Luino, che espresse molteplici professionisti lungo tutto un secolo, anche se il suo membro più famoso si rese celebre come pittore, col soprannome Lui$\mathrm{ni}^{11}$.

Il punto successivo da analizzare è il percorso formativo di tali professionisti, soprattutto di quelli operanti nel contado: dalla documentazione conservata appare chiaro che ad Arezzo la formazione fosse quasi esclusivamente urbana, mentre a Milano essa poteva comportare solo una breve tappa in città, per l'immatricolazione, mentre il resto della carriera poteva tranquillamente svolgersi totalmente nel contado. Fra i notai così formati erano comunque assenti chierici, secondo la tradizione dell'Italia comunale (a Milano vi è tuttavia un caso), ed essi erano invariabilmente di nomina non comunale, quasi sempre imperiale a Milano, qualche volta anche pontificia ad Arezzo ${ }^{12}$.

Quello che è certo è che tali professionisti poi finissero invariabilmente per entrare nel collegio urbano, magari solo formalmente, in entrambe le città. Ma qui si sfiora un altro grosso problema, quello del ruolo e della forza di tali consessi, che certo non erano ininfluenti per lo svolgimento delle singole carriere. In realtà mancano quasi studi in merito prima del Quattrocento (ad Arezzo ve ne è uno per il Trecento), per cui per il periodo precedente si è costretti - soprattutto per il Duecento - a procedere per ipotesi se non si vuole arbitrariamente retrodatare la situazione posteriore. Il collegio milanese era indubbiamente molto forte e in grado di far valere le proprie ragioni di fronte a chiunque, mentre ad Arezzo era sottoposto al comune, anche se talvolta poteva godere di una notevole autonomia. Ma si tratta di congetture, almeno per il Duecento, come abbiamo detto. Si fonda invece sull'osservazione diretta della documentazione la descrizione del curriculum formativo notarile, che è però da considerare pienamente canonizzato solo nel XIV secolo inoltrato. A Milano tale percorso prevedeva invariabilmente tre tappe: pronotaio, notaio (laudatus ad omnia) e giudice ordinario. La prima qualifica permetteva una funzione di semplice convalida degli atti rogati da altri; la seconda configurava il notariato effettivamente operante in piena autonomia, mentre la terza offriva soprattutto un titolo onorifico, con qualche compito di maggior rilievo a lei affidata in via preferenziale (ma senza una vera esclusività: altro, infatti, erano i giudici del comune effettivamente attivi come tali). Come è ovvio non tutti i professionisti compivano per intero il percorso, fermandosi ai gradini precedenti secondo le loro convenienze. Ad Arezzo invece il curriculum consisteva nella semplice qualifica di notaio, dato che non si ha traccia di pronotai e la carica di giudice ordinario appare fondamentalmente slegata dalla professione notarile ${ }^{13}$.

11 Per Arezzo la bibliografia ha sostanzialmente tralasciato questo punto, ma ci sono degli ottimi spunti in Barbagli, 2011. Per Milano invece si può contare sul censimento specifico di cui si è detto: Lunari, Sala, Scharf (eds.), 2009.

12 Ibidem, per Milano. Per Arezzo Scharf, 2018b.

13 Vedi lo studio di Barbagli per Arezzo: Barbagli, 2011. Per Milano, oltre alla panoramica 


\section{Il formulario notarile nelle due città}

È opportuno concludere entrando nel dettaglio, nel vivo delle pratiche notarili osservate nelle due città. Di evidenza palmare il fatto che il formulario in uso sia molto simile e con scarse evoluzioni cronologiche. Qualche differenza esiste, ma si tratta di cose minori e che non mutano sensibilmente il quadro. Queste minute differenze si manifestano soprattutto nel formulario, e possono offrire qualche spunto di riflessione. Si offre qui un duplice esempio, che può essere un buon punto di partenza per future elucubrazioni. A Milano, almeno fino a metà del Trecento, è frequente in tutti i contratti che prevedevano una consegna in numerario una singolare clausola che imponeva il pagamento solo in contante, ma comunque mai facendo uso delle carte di debito emesse dal comune ambrosiano, sorta di obbligazioni ante litteram che avrebbero dovuto accrescere le disponibilità finanziare del comune stesso. Niente di tutto ciò compare ad Arezzo, e il fatto si spiega con la politica monetaria di minore cabotaggio seguita dalla città di San Donato, che non aveva avuto bisogno di simili emissioni. In compenso a partire dalla metà del Duecento in quest'ultima città è quasi onnipresente in simili contratti la clausola sulla guarentigia, norma statutaria che permetteva di rendere immediatamente esecutive le obbligazioni private, senza bisogno di ulteriori interventi della giustizia comunale. Pur non essendo assente dagli atti milanesi, la detta clausola è in essi molto rara, e dimostra dunque una diversa politica del comune ambrosiano nei confronti dei debiti privati, che si volevano evidentemente tenere sotto controllo ${ }^{14}$.

Come abbiamo detto simili osservazioni potrebbero essere ripetute in abbondanza, ma esse non muterebbero sensibilmente il quadro generale fin qui tratteggiato, invero piuttosto chiaro. In conclusione il parallelo mostrato ha evidenziato numerose differenze di minor portata, ma inserite in un quadro generale comune, idealmente estensibile anche ad altre realtà, e ciò giustifica dunque un notariato che, pur agendo localmente, non doveva trovare difficile inserirsi nelle pratiche di un nuovo territorio, non troppo dissimili dalle proprie, qualora ne avesse avuto necessità.

offerta da Liva, 1979, si vedano le introduzioni a Lunari, Sala, Scharf (eds.), 2009, e BelIoni, Lunari (eds.), 2004. Sul ruolo dei pronotai trecenteschi, esito di un lungo consolidamento, vedi Perelli Cippo, 1982.

${ }^{14}$ I due problemi hanno goduto di qualche fortuna storiografica, anche se non sono certo al centro di specifici studi recenti: vedi Biscaro, 1928, e ora naturalmente Grillo, 2001; Paoli, 1882; Campitelli, 1970. 


\section{Bibliografia}

Barbagli A., 2011: I/ notariato ad Arezzo tra Medioevo ed età moderna, Milano, Giuffrè

Barlucchi A., 2013: I centri minori delle conche appenniniche (Casentino e Alta Valtiberina), in G. Pinto, P. Pirillo (eds.), I centri minori della Toscana nel Medioevo, atti del convegno (Figline Valdarno 2009), Firenze, Olschki, pp. 57-95

Baroni M.F. (ed.), 1976: Gli atti del Comune di Milano nel secolo XIII. Vol. 1: 12171250, Milano, s.ed.

Baroni M.F., 1978: II notaio milanese e la redazione del documento tra il 1115 e il 1250, in Felix olim Lombardia. Studi di storia padana in onore di Giuseppe Martini, Milano, s.ed., pp. 5-25

Baroni M.F. (ed.), 1992: Gli atti del Comune di Milano nel secolo XIII. vol. 3: 12771300, 2 tt., Alessandria, Ferraris

Baroni M.F. (ed.), 1997-1998: Gli atti del Comune di Milano nel secolo XIII. Vol. 4: Appendice: 1176-sec. XIII. Indice dei nomi di persona e di luogo, elenco dei documenti editi nell'opera, Alessandria, Ferraris

Baroni M.F., Perelli Cippo R. (eds.), 1982-1988: Gli atti del Comune di Milano nel secolo XIII. vol. 2: 1251-1276, 3 tt., Alessandria, Ferraris

Bedina A., 1992: II protocollo notarile di Francescolo Oldoni notaio di Milano (1390-1393), in "Studi di Storia Medievale e Diplomatica", XII-XIII, pp. 71-90

Belloni C., Lunari M. (eds.), 2004: I notai della curia arcivescovile di Milano: secoli $X I V-X V$, coordinamento di G. Chittolini, Roma, Ministero per i beni e le attività culturali, Direzione generale per gli archivi

Biscaro G., 1928: Gli estimi del Comune di Milano nel secolo XIII, in "Archivio Storico Lombardo", LV, pp. 343-495

Campitelli A., 1970: Precetto di guarentigia e formule di esecuzione parata nei documenti italiani del secolo XIII, Milano, Giuffrè

Canaccini F., 2002: Città e contado ad Arezzo al tempo di Guido Tarlati negli atti notarili di ser Feo di Rodolfo, in "Annali Aretini", X, pp. 155-170

Castelnuovo G., 2007: La geografia amministrativa del contado milanese nel secolo XIII, in "Nuova Rivista Storica", XCl, pp. 233-58

Cereghini B., 1992: L'archivio notarile, in G. Cagliari Poli (ed.) L'Archivio di Stato di Milano, Firenze, Nardini

Cherubini G., 2001: Le attività economiche degli aretini tra XIII e XIV secolo, in "Quaderni Medievali", XXVI, 52, pp. 19-63

Chiappa Mauri L., 1993: Gerarchie insediative e distrettuazione rurale nella Lombardia del secolo XIV, in L. Chiappa Mauri, L. De Angelis Cappabianca, P. Mainoni (eds.), L'eta dei Visconti: il dominio di Milano fra XIII e XV secolo, 
Milano, La storia, pp. 269-301

Chiappa Mauri L. (ed.), 2004: Contado e città in dialogo. Comuni urbani e comunità rurali nella Lombardia medievale, Bologna, Cisalpino

Chittolini G., 1994: «Episcopalis curie notarius». Cenni sui notai di curie vescovili nell'Italia centro-settentrionale alla fine del Medioevo, in Società, Istituzioni, Spiritualità. Studi in onore di Cinzio Violante, Spoleto, CISAM, pp. 221-32

Chittolini G., Willoweit D. (eds.), 1994: L'organizzazione del territorio in Italia e in Germania: secoli XIII-XIV, Bologna, II Mulino

Città e campagna nei secoli altomedievali, 2009: Atti della settimana di studio (Spoleto 2008), Spoleto, CISAM

Del Bo B., 2010: Banca e politica a Milano a metà Quattrocento, Roma, Viella

Delumeau J.P., 1996: Arezzo 715-1230: espace et societè, Roma, École Française de Rome

Dini B., 1984: Arezzo intorno al 1400. Produzioni e mercato, Arezzo, Camera di Commercio

Dini B., 1995: La presenza dei valligiani sul mercato di Arezzo, in G. Renzi (ed.), La Valtiberina Lorenzo e i Medici, Firenze, Olschki, pp. 183-200

Fissore G.G., 1978: La diplomatica del documento comunale fra notariato $e$ cancelleria. Gli atti del comune di Asti e la loro collocazione nel quadro dei rapporti fra notai e potere, in "Studi Medievali", s. 3 XIX, fasc. I, pp. 211-44

Fissore G.G., 1989: Alle origini del documento comunale. I rapporti fra i notai e l'istituzione, in Civiltà comunale: Libro, scrittura, documento, in Atti del Congresso, Genova 8-11 novembre 1988, in "Atti della Società Ligure di Storia Patria", n.s. XXIX (CIII), fasc. II, pp. 99-128

Fois L. (ed.), 2008: Le pergamene duecentesche di Santo Stefano di Vimercate conservate presso l'Archivio di Stato di Milano (1201-1234), Milano, s.ed. (Pergamene milanesi dei secoli XII-XIII, 21)

Fois L. (ed.), 2010: Le pergamene duecentesche di Santo Stefano di Vimercate (1234-1273), Milano, Edizioni Biblioteca Francescana (Studi di storia del Cristianesimo e delle Chiese cristiane. Fonti e documenti, 4)

Fois L. (ed.), 2012: Le pergamene duecentesche di Santo Stefano di Vimercate (1273-1300), Milano, Edizioni Biblioteca Francescana (Studi di storia del Cristianesimo e delle Chiese cristiane. Fonti e documenti, 7)

Franceschi F., 2009: Spunti per una storia dei rapporti economici tra città e campagna in alcuni notai aretini del Trecento, in R. Mucciarelli, G. Piccinni, G. Pinto (eds.), La costruzione del dominio cittadino sulle campagne. Italia centrosettentrionale, secoli XII-XIV, atti del convegno (Siena 2004), Siena, Protagon, pp. 651-67

Gamberini A., 2004: II contado di Milano nel Trecento. Aspetti politici e 
giurisdizionali, in L. Chiappa Mauri (ed.), Contado e città in dialogo. Comuni urbani e comunità rurali nella Lombardia medievale, Bologna, Cisalpino, pp. 83-137

Ginatempo M., 1997: Le città italiane, XIV-XV secolo, in Poderes pùblicos en la Europa Medieval: Principados, Reinos y Coronas, Actas de la XXIII Semana de Estdios Medievales de Estella, Pamplona, Gobierno de Navarra, pp. 149-209

Grillo P., 2001: L'introduzione dell'estimo e la politica fiscale del comune di Milano alla metà del secolo XIII (1240-1260), in P. Mainoni (ed.), Politiche finanziarie e fiscali nell'Italia settentrionale (secoli XIII - XV), Milano, Unicopli, pp. 11-37

Guzzi C., Mainoni P., Zelioli Pini F. (eds.), 2012: Lecco viscontea: gli atti dei notai di Lecco e del suo territorio (1343-1409), Annone Brianza, Cattaneo

Jones Ph., 1997: The Italian City States, from Commune to Signoria, Oxford, Clarendon Press

Liva A., 1979: Notariato e documento notarile a Milano dall'alto Medioevo alla fine del Settecento, Roma, Consiglio Nazionale del Notariato

Lucioni A., Scharf G.P.G. (eds.), 2021: Le Fonti duecentesche per la storia della provincia di Varese. Documenti degli archivi minori, Milano, Vita e Pensiero

Lunari M., Sala M.P., Scharf G.P.G. (eds.), 2009: Notai del contado milanese in epoca viscontea (1347-1447), Milano, Unicopli

Mangini M.L., 2011a: Le scritture duecentesche in quaterno dei notai al servizio della Chiesa Ambrosiana, in "Studi Medievali", LII/1, pp. 1-49

Mangini M.L., 2011b: /l più antico quaternus imbreviaturarum dell'Archivio Notarile di Milano (1290-1294). Un unicum per il Duecento milanese?, in "Annuario dell'Archivio di Stato di Milano", n.s. I, pp. 87-106

Mangini M.L. (ed.), 2011c: I quaterni imbreviaturarum di Giovannibello Bentevoglio de Vaprio, Milano, Edizioni Biblioteca Francescana

Mangini M.L., 2012: Nuovi itinerari di ricerca sui protocolli notarili milanesi del secolo XIII. Un frammento del quaternus di Giacomo, notaio in Milano (1276), in P. Cherubini, G. Nicolaj (eds.), Sit liber gratus, quem servulus est operatus: studi in onore di Alessandro Pratesi per il suo XC compleanno, Città del Vaticano, Scuola vaticana di paleografia, diplomatica e archivistica, pp. 549-63

Menant F., Redon O. (eds.), 2004: Notaires et crédit dans l'Occident Méditerranéen Médiéval, Rome, École Française de Rome

Merati P. (ed.), 2005: Le carte della chiesa di Santa Maria del Monte di Velate, vol. I: 922-1170, con note introduttive di C. Storti e M.F. Baroni, Varese, IUP

Merati P. (ed.), 2006: Le carte della chiesa di Santa Maria del Monte di Velate, vol. II: 1171-1190, Varese, IUP

Merati P. (ed.), 2009: Le carte della chiesa di Santa Maria del Monte di Velate, vol. III: 1191 - 1200, Varese, IUP 
Mucciarelli R., Piccinni G., Pinto G. (eds.), 2009: La costruzione del dominio cittadino sulle campagne. Italia centro-settentrionale, secoli XII-XIV, atti del convegno (Siena 2004), Siena, Protagon

Paoli C., 1882: Miscellanea di paleografia e diplomatica. VIII. Due statuti del secolo XIII sul comandamento della guarentigia, in "Archivio Storico Italiano", s. IV, t. X, pp. 250-8

Pasqui U., 1916: Documenti per la storia della città di Arezzo nel Medio Evo, II, Firenze, Deputazione di Storia Patria per la Toscana

Perelli Cippo R. (ed.), 1976: Regesto di S. Maria di Monte Velate (sec. XIII), Firenze, La Nuova Italia

Perelli Cippo R., 1982: "Notarii" $e$ "secundi notarii" a Milano nel Duecento, in "Nuova Rivista Storica", LXVI, f. III, pp. 594-98

Pieri S., 2002: La visita pastorale di Guglielmo degli Ubertini (1257 - 1258), in «Annali Aretini», X, pp. 61-108, riedito in S. Pieri, C. Volpi (eds.), Visite Pastorali dal 1257 al 1516, Diocesi di Arezzo-Cortona-Sansepolcro, Fiesole, Servizio Editoriale Fiesolano, 2006, pp. 11-59

Pini A.I., 1987: Dal comune città-stato al comune ente amministrativo, in Id., Città, comuni e corporazioni nel medioevo italiano, Bologna, C.L.U.E.B., pp. 59-218

Pinto G., 1999: Produzione e traffici nell'aretino nei secoli XIII e XIV. Aspetti e problemi, in "Atti e Memorie dell'Accademia Petrarca di Lettere, Arti e Scienze", n. s., LXI, pp. 223-236

Pinto G., Tanzini L., Tognetti S. (eds.), 2018: Notariorum itinera. Notai toscani del basso Medioevo tra routine, mobilità e specializzazione, Firenze, Olschki

Riboldi E., 1904: I contadi rurali del milanese (sec. IX - XII), in "Archivio Storico Lombardo", XXXI, fasc. I, pp. 15-74, fasc. II, pp. 240-302

Scharf G.P.G., 2013: Potere e società ad Arezzo nel XIII secolo (1214-1312), Spoleto, CISAM

Scharf G.P.G., 2015: La distrettuazione minore del contado milanese: pievi e vicariati del Seprio in epoca viscontea-sforzesca, in M.M. Grisoni (ed.), 2015: I Visconti: residenze e territorio. Conoscere per tutelare e valorizzare il paesaggio storico. Atti del convegno I Visconti: Ia famiglia, l'architettura, il territorio, tenuto a Somma Lombardo il 13 ottobre 2013, Livorno, Debatte, pp. 21-25

Scharf G.P.G., 2018a: /l Seprio tardomedievale, strutture e popolamento, in G. Archetti (ed.), Teodolinda. I longobardi all'alba dell'Europa, Atti del Secondo convegno internazionale di studio (Monza, Gazzada, Castelseprio-Torba, Cairate, 2-7 dicembre 2015), Milano-Spoleto, Centro Studi Longobardi-CISAM, 2018, pp. 717-22

Scharf G.P.G., 2018b: I notai aretini fra Due e Trecento, in G. Pinto, L. Tanzini, S. Tognetti (eds.), Notariorum itinera. Notai toscani del basso Medioevo tra routine, mobilità e specializzazione, Firenze, Olschki, pp. 91-98 
Schiaparelli L., Baldasseroni F., Lasinio E. (eds.), 1909, 1914, 1922: Regesto di Camaldoli, voll. II, III, IV, Roma, E. Loescher

Zagni L., 1982: La redazione dei protocolli notarili a Milano nel secolo XIV, in "Studi di Storia Medievale e Diplomatica", VII, pp. 43-53

Zagni L. (ed.), 1992: Le pergamene della basilica di s. Vittore di Varese (899-1202), Milano, s.ed. (Pergamene milanesi dei secoli XII-XIII, 9)

Zagni L. (ed.), 1999: Le pergamene della basilica di S. Vittore di Varese (12041260), vol. II, Milano, s.ed. (Pergamene milanesi dei secoli XII-XIII, 13)

Zagni L. (ed.), 2005: Le pergamene della basilica di S. Vittore di Varese (12611289), vol. III, Milano, s.ed. (Pergamene milanesi dei secoli XII-XIII, 19) 
